Journal of Career Assessment

Volume 2/Number 1/Winter 1994/Pages 29-39

\title{
Convergence and Obfuscation: A Rejoinder to Osipow and to Laplante, Coallier, Sabourin, and Martin
}

\author{
John Schulenberg \\ University of Michigan \\ and \\ Fred W. Vondracek \\ The Pennsylvania State University \\ and \\ Kazuaki Shimizu \\ Kansai University
}

\begin{abstract}
Our previous critique of Martin, Sabourin, Laplante, and Coallier (1991) included 10 serious criticisms of their methods and conclusions regarding the dimensionality of the Career Decision Scale (CDS). Although the quantity of the Laplante, Coallier, Sabourin, and Martin (1994) response was not bad (i.e., they attempted to address 7 of the 10 criticisms), the quality of their response was disappointing because it confused fact with fiction, and included a wealth of extraneous and irrelevant material. Nothing in their response convinced (or even tempted) us to retract any of our original criticisms. Most importantly, the equivalence of their French version of the CDS with the English version was still not demonstrated, our four-factor model was still not disconfirmed, and their model testing procedures remain suspect (e.g., for some undisclosed reason, Laplante et al. (1994) and Martin et al. (1991) are at odds as to whether they accepted their one-factor model). Based on our findings (as well as those of Martin et al. [1991]despite their conclusions, their findings support our model), the fact remains that our four-factor model provided the best fit among the several models tested, indicating firm support for the multidimensionality of the CDS. We found ourselves in substantial agreement with Osipow (1994), and we urge him to refine the CDS by adding dimension-specific items and revising double-barreled items.
\end{abstract}

We appreciated the response of Laplante, Coallier, Sabourin, and Martin (1994) and the comments of Osipow (1994) regarding the dimensionality of the Career Decision Scale (CDS; Osipow, 1987; Osipow, Carney, Winer, Yanico, \& Koschier, 1976). While we may not all be of one mind on the

\footnotetext{
We wish to thank Rainer Silbereisen for helpful comments regarding this manuscript.
}

Published and copyright $\odot 1994$ by Psychological Assessment Resources, Inc. All rights reserved. 
various issues involved, we certainly all share a common mission to understand the origins, processes, and consequences of career indecision among adolescents and adults. Our rejoinder is cast with this common mission in mind, and offered in the spirit of scholarly criticism and debate.

Our critique of Martin, Sabourin, Laplante, and Coallier (1991) (Shimizu, Vondracek, \& Schulenberg, 1994) included several severe and specific criticisms of their methodology and ultimately of their conclusions about the multidimensionality of the CDS. For the purposes of scientific advancement and intellectual exchange, we were awaiting with interest their response to our criticisms. Unfortunately, their response (Laplante et al., 1994) was disappointing in several respects. In particular, they avoided addressing key criticisms by focusing on side issues and putting an obscure spin on some of their previous statements. Before we address our specific concerns with Laplante et al. (1994), we first respond to Osipow's comments.

\section{Response to Osipow: We Agree, and it Could be Better}

\section{Convergence}

The comments of Osipow (1994), senior author of the CDS (Osipow, 1987; Osipow et al., 1976), help place the current methodological issues about the dimensionality of the CDS in the context of historical and practical considerations. We found ourselves in substantial agreement with Osipow on several points, including: that Martin et al.'s (1991) failure to consider cultural and language differences detracts seriously from their conclusions; that both career indecision and the CDS are probably best viewed as multidimensional; and that despite its known flaws, the CDS has remained a robust instrument for nearly 2 decades because of its "clinical" basis and practical value.

\section{Minor Disagreement}

There is only one minor point of disagreement with Osipow. Although the appropriate use of factor analysis may involve some "subjective judgment," we believe it is incorrect to characterize such subjective judgment as "considerable," particularly when one is attempting to determine the similarities of factors across samples or time. The necessity for and benefits of restraining subjective judgments in factor analysis is exemplified in our exploratory and confirmatory factor analyses of the CDS (Shimizu, Vondracek, Schulenberg, \& Hostetler, 1988; Schulenberg, Shimizu, Vondracek, \& Hostetler, 1988). The point here is that very little of our disagreement with Martin et al. (1991) over the dimensionality of the CDS can be attributed to differences in "subjective judgments."

\section{Clarification}

In regard to Osipow's (1994) conclusions, we wish to clarify one of our statements. In discussing the dimensionality of the CDS, we stated "with all due respect to Professor Osipow and his colleagues, it matters little what he originally intended when constructing the CDS" (Shimizu et al., 1994 , p. 8). This statement was strictly in reference to whether Osipow et al. (1976) intended to form one or several dimensions of career indecision, 
and not at all in reference to the authors' intended purpose for constructing the CDS (which, of course, is important for validity purposes).

\section{Revise the CDS}

Our response to the question Osipow (1994) posed in his title regarding the CDS, "How Good Does it Have to be?" is that it could be better in two respects (see also Slaney, 1988, Vondracek, Hostetler, Schulenberg, Shimizu, 1990). First, as Osipow (1994) suggests, the CDS is in need of more items. To the extent that empirical support continues to mount for the existence of four sub-scales (e.g., Chartrand \& Robbins, 1990; also Martin et al., 1991-contrary to their conclusions, their findings support our four-factor model), then additional items should be included to provide more reliable assessment of the sub-scales. At the same time, items that do not tap a single dimension should be deleted.

Secondly, double-barreled items should be revised. The complexity of such items contributes to unreliability, to inflated correlations among the subscales, and to irritability among respondents. Despite the unpublished evidence that the double-barreled wording makes little substantive difference in findings (Damerin, 1981), we believe strongly that the elimination of double-barreled items would make the CDS a better instrument.

Accomplishing these revisions would not necessarily render findings and norms based on the current version obsolete, as Osipow (1994, pp. 15-18) appears to suggest. In our view, the revised version of the CDS should be as similar as possible to the current version in form and content. To the extent that the two versions are documented to be similar, then previous findings and norms will remain useful. The effort to revise the CDS would yield a more psychometrically sound instrument to carry us through the next 2 decades.

\section{Response to Laplante et al.: The Dimensionality of the CDS is an Empirical Issue; Obfuscation Will not Change the Facts}

Our critique (Shimizu et al., 1994) of Martin et al. (1991) included at least 10 separate criticisms of their methods and conclusions. Although the quantity of the Laplante et al. (1994) response was not bad (i.e., they attempted to address 7 of the 10 criticisms), the quality of their response was disappointing because, as we shall demonstrate, it confused fact with fiction, and included a wealth of extraneous and irrelevant material. In the first two sections we consider whether Laplante et al. (1994) adequately addressed our major and more minor criticisms of their work. In the third section we confront a major discrepancy between key statements made by Martin et al. (1991) and Laplante et al. (1994) regarding whether they found their unidimensional model acceptable, a matter central to our criticism of their work. In the fourth section we dismiss remaining fictions and recover buried facts.

\section{Update: Equivalence is Still not Demonstrated, and the Four-Factor Model is Still not Disconfirmed}

Our three primary criticisms of Martin et al. (1991) can be distilled down to two simple questions: (a) Was it demonstrated prior to (or even after) the 
Martin et al. (1991) study that their French version of the CDS was equivalent (i.e., measured the same thing in the same way) to the English version? and (b) Among the models appropriately (or even inappropriately) tested using their data and ours, was there any evidence to disconfirm our four-factor model? Laplante et al. (1994) failed to inspire us to change our original answers: (a) No, and (b) No. Let us elaborate in light of Laplante et al.'s (1994) failed attempt to convince us to retract our criticisms.

Simple Fact 1: It is not possible to compare two quantities unless it is clear that the same measuring device is being used. Despite the rather lengthy response to our criticism about their failure to first establish measurement equivalence between their French version of the CDS and the English version, Laplante et al. (1994) still failed to provide any new evidence to indicate that the two measures are equivalent. Of course, they provide some helpful, albeit superficial, evidence indicating that the two cultures involved are similar in several important respects, and that their French version of the CDS and the English version appear to be related to other constructs in similar ways. Still, similarity in culture and outcomes does not constitute measurement equivalence (nor do speculative intentions regarding the use of IRT), and discussing such matters is a poor substitute for implementing such simple procedures as back-translation. This issue is crucial, for without the a priori (or even post-hoc) demonstration of equivalence, there is little justification for making cross-cultural comparisons of factor models.

Do their factor analyses constitute evidence of equivalence, as Laplante et al. (1994) suggest? According to the logic of model testing, such would be true if they accepted the four-factor model as the best-fitting model, and if they viewed their study as a measurement-equivalence study rather than a model-testing study. That is, one cannot, in a single study, demonstrate measurement equivalence and confirm or disconfirm previous models.

Simple Fact 2: The best-fitting model that is not disconfirmed remains the most plausible approximation of reality. Martin et al. (1991) followed appropriate procedures by specifying a priori, three competing statistically models based on 13 CDS items. Because the three models were not "nested" (e.g., see Schulenberg et al., 1988), it was not possible to directly statistically compare the three models, and thus model comparisons were based on the absolute fit of the models to the data. Their tests of these three models (as well as their 12-item unidimensional model) revealed that the four-factor model provided the best fit to the data based on the criteria they presented. Nevertheless, Martin et al. (1991) rejected the four-factor model in favor of their 12-item unidimensional model. We criticized them for their lapse in judgment and in an exact replication of their models using our data, we again demonstrated that the four-factor model provided the best fit to the data (Shimizu et al., 1994).

Laplante et al.'s (1994) response to our criticism surprised even us. They utterly dismissed all fit indices except for the chi-square test statistic. We admit some appreciation for iconoclastic positions, but theirs is completely contrary to logic and established procedures. For some undisclosed reason, not only are they unwilling to believe our models based on our data, or 
even their models based on our data, they also are unwilling to believe their own models based on their own data. A brief lesson in the basics of model comparison may be needed:

(1) Why use several fit indices? Because none is a perfect representation of all the different aspects of "good fit."

(2) Is one index better than all the others? No; although some tend to be better than others in certain situations, no one index is always better than all others.

(3) Is the chi-square test statistic among the better fit indices? No, because "the well-known problem with this is that chi-square depends directly on the sample size, leading to almost certain rejection of models in large samples even when they differ trivially from perfect fit" (Wheaton, 1988, p. 201).

(4) Were the significant chi-square fit statistics found by Shimizu et al. (1994) due to the relatively large sample size $(N=703)$ ? Probably; had our sample size been about 500, the chi-square for the multidimensional model (with 13 items) would have been non-significant, indicating an acceptable fit to the data.

(5) How can you tell if you have a "good fitting" model? Typically, convergence among the various criteria is the best indicator of a "good fit."

(6) Are there other fit indices that have not yet been presented that might provide new information to help settle the issue? Yes, Bentler (1990) proposed the Comparative Normed Fit Index (CNI) (see also Mulaik, James, Van Alstine, Bennett, Lind, \& Stilwell, 1989) that is not dependent on sample size, in which fit increases as the index approaches 1.00; in our reanalysis, the CNI was $.930, .985$, and .981 , for the unidimensional, multidimensional, and hierarchical models (with 13 items), respectively (see Shimizu et al., 1994).

(7) So, based on the various fit indices, what is the "bottom line"? The four factor model is the best fitting model among all models tested, and it provides a very good fit to the data.

Simple Fact 3: Deleting an item solely because it causes a poor fit in one, but not all models, and then re-estimating the one model does not constitute acceptable model testing procedures. Our third major criticism of Martin et al. (1991) was that they arbitrarily deleted an item (\#18) in an effort to provide a better fit of the unidimensional model to the data. This is more than simply "overfitting" the model to the given sample data. Deleting an item because of its lack of fit in one model and then comparing that model to other models that included the offending item is far from acceptable model testing procedures. Laplante et al.'s (1994) response to our criticism seems to center around the fictitious claim that they (Martin et al., 1991) also reported the findings of the four-factor model that excluded item 18 . They deny that their exclusion of item 18 was arbitrary, although if one assumes that arbitrary means "for no justifiable reason," then their denial has no basis. The fact that item 18 proved important in our reanalysis of our 
data with their models underscores the perils of arbitrarily excluding items. The irony here is that even with the exclusion of item 18 , the unidimensional model still provided a worse fit than our four-factor model (including item 18) to their data and our data.

\section{Seven Other Lessons in Model Testing}

By our count, Laplante et al. (1994) are now 0 for 3 in terms of adequately addressing our criticisms. How did they do with the remaining 7 criticisms?

\section{Factor Correlations do not Constitute Disconfirming Evidence}

We criticized Martin et al. (1991) for using factor correlations as important evidence for rejecting the four-factor model. Laplante et al. (1994) deny that factor correlations were a primary reason for rejecting the four-factor model, and indicate that there were other (unspecified) reasons for their rejection. Neither part of their response is defensible, given their (Martin et al., 1991) original statements. Let us reiterate here: Criteria for model confirmation or disconfirmation typically do not include factor correlations. For example, even Sabourin, Lussier, Laplante, and Wright (1990) accepted multidimensional models of an instrument measuring dyadic adjustment with factor correlations ranging from .61 to .98 .

\section{Does not Equal 13 or 12}

We criticized Martin et al. (1991) for advocating the use of the CDS total score (summary of all 16 items) based on their (erroneous) acceptance of a unidimensional model based on 12 items. Laplante et al. (1994) failed to address this criticism. They do, however, indicate that they did test models with all 16 items, but they failed to describe these models or to provide fit indices. Given the poor fit of the 13-item unidimensional model, we feel quite justified in assuming that the 16-item unidimensional model provided a very poor fit to the data.

\section{Initial Conceptualizations Matter Less Than Current Empirical Support}

We criticized Martin et al. (1991) for basing their support of the unidimensional model over the four-factor model on their (incorrect) view that the CDS was designed to tap a single dimension (see Winer, 1992, for a review of the history of the CDS). We indicated that the better judge of the dimensionality of the CDS is current empirical evidence, the preponderance of which (including Martin et al., 1991) supports our four-factor model. Although Laplante et al.'s (1994) response is not entirely clear, they seem to be advocating an ascientific approach to model selection. Ignoring the weight of the scientific evidence and issuing a declaration that the CDS is unidimensional is no more acceptable than trying to establish this fiction by editorializing about it (e.g., Tinsley, 1992).

\section{Examine the Number of Factors Indicated by the Data}

We criticized Martin et al. (1991) for failing to consider the number of factors indicated by the data (e.g., according to the scree test). This was a crucial omission, for whenever one is attempting to specify the dimensional structure of a set of data, it is wise to see what the data suggest. Laplante et al. (1994) failed to respond to this criticism. 


\section{Document the Nonnormality of the Data}

We criticized Martin et al. (1991) for failing to present evidence supporting their decision to use correlation matrices and estimation procedures that did not assume multivariate normality. Our point was that there are important trade-offs for abandoning the use of variance-covariance matrices and maximum likelihood estimation procedures, and that such abandonment should occur only when necessary. We then provided evidence indicating that our data were probably not sufficiently nonnormal to justify such abandonment (although to replicate their methods we did anyway). Laplante et al. (1994) failed to respond to this criticism.

\section{CDS Items Cannot be Both Ordinal and not Ordinal}

We expressed some sympathy for Martin et al. (1991) for getting themselves into the position of arguing, in the same paper, that the CDS items are and are not ordinal. Laplante et al. (1994) responded to our concerns by essentially stating that they never expected anyone to point out the apparent contradiction.

\section{The Hoofs Still do not Belong to Zebras}

We criticized Martin et al. (1991) for excluding obvious explanations for the failure of their hierarchical model, and for, instead, pursuing speculative explanations. Laplante et al.'s (1994) response seems to be one of resignation: "In simple words, the hierarchical model is just another possible dimensional structure that doesn't fit the CDS items" (pp. 22-23). We note that the hierarchical model provided a good fit to our data, although not as good a fit as the four-factor model.

\section{Was the Unidimensional Model Acceptable?}

\section{"Yes" (Martin et al., 1991)}

According to Martin et al. (1991) the results of their factor analyses of the CDS indicated that "a unidimensional model was the most parsimonious" (p. 187), "the unidimensional model appeared to be well adjusted to the data" (p. 193), and "since results showed the adequacy of the unidimensional model, use of the CDS total score should be promoted" (p. 195). We trust that the reader will judge for herself or himself that these statements are not taken out of context and are representative of the tone of the Martin et al. (1991) conclusions. Therefore, one could reasonably assume, as we did, that Martin et al. (1991) concluded that the unidimensional model is an acceptable model, and is preferred over the multidimensional model. We make a point in our critique (Shimizu et al., 1994) of disagreeing with their conclusion because the fit indices they presented indicated that the multidimensional model fit better than the unidimensional model.

\section{“No" (Laplante et al., 1994)}

In their response to us, Laplante et al. (1994) deny their original conclusion. They state that "contrary to what Shimizu et al. (1988) seem to understand, we do not conclude that the CDS is unidimensional" (p. 26), that "neither the unidimensional nor the multidimensional model can account for 
the correlation structure that exists among the CDS items. It is from these results that we recommended that the CDS be regarded as an instrument measuring only one characteristic" (p. 21), and that "we concluded that the CDS has no clear dimensional structure and recommended the use of the total score" (p. 19). We note here that the existence of even one-dimension (or characteristic) presumes a dimensional structure.

\section{Why the Curious Turnabout?}

It seems odd to us that they would promote the superiority of the one-factor model in their original article, and then dismiss it in the their response. We can only guess the reason behind the turnabout, and at least two possibilities occurred to us. First, perhaps their research team was not of one mind about the issue of dimensionality. On purely logical grounds, they were better off with their original conclusion. That is, it is much more logically defensible to advocate the use of a total scale score when one concludes that the scale is composed of a single dimension. Otherwise, one is in the unenviable position of suggesting that the scale is and is not unidimensional. Of course, the statements listed in the previous paragraph illustrate that this is exactly the position that Laplante et al. (1994) are in, although they seem oblivious to the logical peril of their position.

A second possibility that may explain the turnabout is that once Laplante et al. (1994) realized-perhaps at our urging-that there was no empirical justification for accepting the unidimensional model over the multidimensional model, they took the only possible route that would permit them to still disregard the multidimensional model. That is, by asserting that none of the models provided an adequate fit to the data, it is possible to claim that it does not matter that the multidimensional model provided a better fit than the unidimensional model. Of course, this leaves them in the illogical position just described, and it does nothing to change the reality that the multidimensional model was acceptable and provided a better fit than the unidimensional model.

\section{Dismissing Remaining Fictions, Recovering Important Facts}

There are several additional points in Laplante et al.'s (1994) response that are closer to fiction than fact. First, in our confirmatory factor analyses, we used a variance-covariance matrix, rather than a correlation matrix (as Laplante et al., 1994, indicated). This is an important difference, for not only does this constitute correct procedure given the LISREL algorithms (Joreskog \& Sorbom, 1988, 1989), it is an essential procedure when comparing two or more groups that may differ in observed or latent construct variances (see Schulenberg et al., 1988, for additional discussion). Indeed, the questionable decision by Martin et al. (1991) to use polyserial correlations assumes equivalent variances between their sample and ours, and precludes the use of appropriate multigroup comparison procedures.

In attempting to defend their arbitrary exclusion of item 18, Laplante et al. (1994) described us as "authors who consider their results of the factor analysis of 13 of the 16 items of the CDS as solid proof of the existence of four dimensions of career indecision!" (p. 22). Although they did not directly 
say it, our powerful abilities of intuition revealed to us that they were implying that our exclusion of the 3 items was arbitrary. A reading of the clear rationale and procedures for excluding the 3 items in our previous articles (Schulenberg et al., 1988; Shimizu et al., 1988) should cure them of this misapprehension. As for "solid proof" (see also the last sentence in Laplante et al., 1994), we are social-behavioral scientists who humbly reserve that term for physicists and mathematicians.

Laplante et al. (1994) indicated that we suggested that they leave their factor intercorrelation matrix unconstrained in their attempt to find one higher-order factor. This is fiction. The fact is that we suggested "perhaps there exist more than one higher-order factor" (Shimizu et al., 1994, p. 9). That is, contrary to Laplante et al.'s (1994) apparent understanding of hierarchical confirmatory factor analysis, one can model more than one higher-order factor. Furthermore, contrary to Laplante et al.'s (1994) claim that we did not specify whether our hierarchical model included constraints on the factor correlations, we clearly stated on page 10 that the hierarchical model included "a second-order factor that would account for the correlations observed among the four first-order factors."

Finally, Laplante et al. (1994) stated that our primary motivation for supporting a multidimensional conception of the CDS is "by considerations of a clinical nature" (p. 24). This is fiction, and the logic that led them to this conclusion escapes us. We have presented a detailed review of the empirical basis for favoring the four-factor model. Their denial of the scientific basis of our preference for this model is consistent, however, with their denial that other investigators have found support for this model. For example, they claimed that Chartrand and Robbins (1990) found no support for our four-factor model. In fact, using a sample of college students, Chartrand and Robbins specifically investigated the relative efficacy of the CDS total scale score and subscales derived from our four-factor model and concluded that "Results supported the use of individual subscales to identify antecedents of career indecision" (p. 166). It is impossible to be more straightforward and Laplante et al.'s (1994) refusal to acknowledge the reported facts can only be explained on the basis of some (undisclosed) ideological basis.

\section{Conclusions}

We want to emphasize that any consumer of the interchanges in this issue of JCA would likely find it useful to read the original articles, and judge the issues according to the data presented and the soundness of the various conceptual and methodological perspectives that have been developed regarding the dimensionality of the CDS. Based on our findings, as well as those of Martin et al. (1991), we remain satisfied that our four-factor model provides the best fit among the models tested. We will maintain this position until new research, employing appropriate samples and appropriate methodology, persuades us to revise our position. It is important, however, to keep in mind that our work has focused on only a narrow aspect of career indecision, namely the factor structure of the CDS among adolescents. Future studies should consider the possibility that the dimensionality of the CDS is given to developmental variation (e.g., see Schulenberg et al., 1988). 
Furthermore, in one area of agreement with Laplante et al. (1994), we believe that future research should include a pattern-centered approach (e.g., cluster analysis) to investigate different types of career indecision (see Savickas \& Jarjoura, 1991, for an extensive discussion of this issue). Of course, we emphasize the need for a revised CDS.

Our increased understanding of career indecision also requires the incorporation of these measurement concerns into conceptual and practical considerations. One area that deserves more attention is the integration of empirical research and current theoretical formulations of career decision-making and indecision (e.g., Gati, 1986; Harren, 1979). Also requiring further attention is the area of clinical and counseling applications. For example, Callanan and Greenhaus (1992) and Vondracek (1993) have demonstrated the possible benefits of differential intervention depending on the type of indecision manifested by the individual. Finally, more attention should be given to understanding the social context that creates largely negative connotations for the state of being undecided (Krumboltz, 1992). Positive attributes associated with this state, such as the excitement of exploration, should receive greater attention.

We hope our exchanges with Martin et al. (1991), Laplante et al. (1994), and Osipow (1994) have illuminated the complexities involved in measuring career indecision. We appreciated the opportunity to be a part of this dialogue.

\section{References}

Bentler, P. M. (1990). Comparative fit indexes in structural models. Psychological Bulletin, $107,238-246$

Callanan, G. A., \& Greenhaus, J. H. (1992). The career indecision of managers and professionals: An examination of multiple subtypes. Journal of Vocational Behavior, 41, $212-231$.

Chartrand, J. M., \& Robbins, S. B. (1990). Using multidimensional career decision instruments to assess career decidedness and implementation. Career Development Quarterly, 39(2), 166-177.

Damerin, F. (1981). [Comparison of the original Career Decision Scale and a shorter, experimental version.] Unpublished raw data.

Gati, I. (1986). Making career decisions-A sequential elimination approach. Journal of Counseling Psychology, 33, 408-417.

Joreskog, K. G., \& Sorbom, M. (1988). PRELIS: A program for multivariate data screening and data summarization. A preprocessor for LISREL (2nd ed.). Mooresville, IN: Scientific Software, Inc.

Joreskog, K. G., \& Sorbom, M. (1989). LISREL 7: User's reference guide. Mooresville, IN: Scientific Software, Inc.

Harren, V. A. (1979). A model of career decision making for college students. Journal of Vocational Behavior, 14, 119-133.

Krumboltz, J. D. (1992). Comment: The wisdom of indecision. Journal of Vocational Behavior, $41,239-244$.

Laplante, B., Coallier, J., Sabourin, S., \& Martin, F. (1994). Dimensionality of the Career Decision Scale: Methodological, cross-cultural, and clinical issues. Journal of Career Assessment, 2(1), 19-28.

Martin, F., Sabourin, S., Laplante, B., \& Coallier, J. C. (1991). Diffusion, support, approach, and external barriers as distinct theoretical dimensions of the Career Decision Scale: Disconfirming evidence? Journal of Vocational Behavior, 38, 187-197. 
Mulaik, S. A., James, L. R., Van Alstine, J., Bennett, N., Lind, S., \& Stilwell, D. (1989). Evaluation of goodness-of-fit indices for structural equation models. Psychological Bulletin, $105,430-445$.

Osipow, S. H. (1987). Manual for the Career Decision Scale (rev. ed.), Odessa, FL: Psychological Assessment Resources.

Osipow, S. H. (1994). The Career Decision Scale: How good does it have to be? Journal of Career Assessment, 2(1), 15-18.

Osipow, S. H., Carney, C. G., Winer, J. L., Yanico, B. J., \& Koschier, M. (1976b). The Career Decision Scale (3rd rev. ed.). Columbus, OH: Marathon Consulting and Press.

Sabourin, S., Lussier, Y., Laplante, B., \& Wright, J. (1990). Unidimensional and multidimensional models of dyadic adjustment: A hierarchical reconciliation. Journal of Counseling and Clinical Psychology, 2, 333-337.

Savickas, M. L., \& Jarjoura, D. (1991). The Career Decision Scale as a type indicator. Journal of Counseling Psychology, 38, 85-90.

Schulenberg, J. E., Shimizu, K., Vondracek, F. W., \& Hostetler, M. (1988). Factorial invariance of career indecision dimensions across junior high and high-school males and females. Journal of Vocational Behavior, 33, 63-81.

Shimizu, K., Vondracek, F. W., \& Schulenberg, J. (1994). Unidimensionality versus multidimensionality of the Career Decision Scale: A critique of Martin, Sabourin, Laplante, and Coallier. Journal of Career Assessment, 2(1), 1-14.

Shimizu, K., Vondracek, F. W., Schulenberg, J. E., \& Hostetler, M. (1988). The factor structure of the Career Decision Scale: Similarities across selected studies. Journal of Vocational Behavior, 32, 213-225.

Slaney, R. B. (1988). The assessment of career decision making. In W. B. Walsh \& S. H. Osipow (Eds.), Career decision making (pp. 33-76). Hillsdale, NJ: Erlbaum.

Tinsley, H. E. A. (1992). Career decision making and career indecision [Editorial]. Journal of Vocational Behavior, 41, 209-211.

Vondracek, F. W. (1993, August). Improving the practice of career intervention through the differential diagnosis and treatment of career indecision. Paper presented at the Third International Symposium on Career Development, Toronto, Canada.

Vondracek, F. W., Hostetler, M., Schulenberg, J. E., \& Shimizu, K. (1990). Dimensions of career indecision. Journal of Counseling Psychology, 37(1), 98-106.

Wheaton, B. (1988). Assessment of fit in overidentified models with latent variables. In J. S. Long (Ed.), Common problems/proper solutions: Avoiding error in quantitative research (pp. 193-225). Newbury Park, CA: Sage. 